\title{
Using the Stokes $V$ widths of Fe I lines for diagnostics of the intrinsic solar photospheric magnetic field
}

\author{
M. Gordovskyy ${ }^{1}$, S. Shelyag ${ }^{2}$, P. K. Browning ${ }^{1}$, and V. G. Lozitsky ${ }^{3}$ \\ 1 Jodrell Bank Centre for Astrophysics, University of Manchester, Manchester M13 9PL, UK \\ e-mail: mykola.gordovskyy@manchester.ac.uk \\ ${ }^{2}$ Faculty of Science, Engineering and Built Environment, Deakin University, Melbourne, VIC 3125, Australia \\ 3 Astronomical Observatory of the Taras Shevchenko National University of Kyiv, Observatorna 3, Kyiv 04053, Ukraine
}

Received 30 October 2019 / Accepted 3 December 2019

\begin{abstract}
Aims. The goal of this study is to explore a novel method for the solar photospheric magnetic field diagnostics using Stokes $V$ widths of different magnetosensitive Fe I spectral lines.

Methods. We calculate Stokes $I$ and $V$ profiles of several Fe I lines based on a one-dimensional photospheric model VAL C using the NICOLE radiative transfer code. These profiles are used to produce calibration curves linking the intrinsic magnetic field values with the widths of blue peaks of Stokes $V$ profiles. The obtained calibration curves are then tested using the Stokes profiles calculated for more realistic photospheric models based on magnetohydrodynamic of magneto-convection.

Results. It is shown that the developed Stokes $V$ widths method can be used with various optical and near-infrared lines. Out of six lines considered in this study, Fe I 6301 line appears to be the most effective: it is sensitive to fields over $\sim 200 \mathrm{G}$ and does not show any saturation up to $\sim 2 \mathrm{kG}$. Other lines considered can also be used for the photospheric field diagnostics with this method, however, only in narrower field value ranges, typically from about $100 \mathrm{G}$ to $700-1000 \mathrm{G}$.

Conclusions. The developed method can be a useful alternative to the classical magnetic line ratio method, particularly when the choice of lines is limited.
\end{abstract}

Key words. Sun: magnetic fields - Sun: photosphere - techniques: imaging spectroscopy

\section{Introduction}

Observations show that the solar photospheric magnetic field is inhomogeneous at spatial scales as short as $10 \mathrm{~km}$, which are not resolved in most optical solar observations. Various observational and computational studies show that the photospheric magnetic flux is formed by small-scale magnetic fluxtubes with field strength of a few $\mathrm{kG}$ surrounded embedded into a much weaker $(\sim 10$ 100 G) ambient field (see Frazier \& Stenflo 1972; Solanki 1993; De Wijn et al. 2009; Stenflo 2011; Bellot Rubio \& Orozco Suárez 2019, for review).

The photospheric magnetic field is normally measured using the Zeeman effect, through Zeeman splitting of the sigmacomponents of spectral lines or through the amplitudes of Stokes $V$ profiles. In the case of spatially-uniform magnetic fields, the measured values represent the longitudinal component of the field vector. However, when the magnetic field is not uniform on the scales not resolved by the instrument, the measured values also depend on the magnetic filling factor, the magnetic field gradient along the line-of-sight (LOS) and other factors which affect the profiles of spectral lines. In the first approximation, known as the two-component model with zero ambient field, the observed field values $B_{\text {obs }}$ are determined by the actual (or intrinsic) magnetic field, corresponding to the small-scale fluxtubes $B_{\text {real }}$, and the filling factor $\alpha$ :

$B_{\text {obs }}=\alpha B_{\text {real }}$.

Knowing the photospheric magnetic field is essential in many areas of solar physics, from observational helioseismology to the coronal magnetic field reconstruction. Some applications are not sensitive to the small-scale structure of the magnetic field. For instance, force-free coronal magnetic field reconstruction is normally sensitive only to the large-scale distribution of photospheric magnetic field (e.g. Wiegelmann \& Sakurai 2012). At the same time, there are characteristics which are very sensitive to the magnetic field filling factor. For instance, the Poynting flux and the magnetic energy density at the photosphere are often used for observational studies of the coronal heating and coronal magnetic field stability (Welsch 2014; Jang 2016). These values are proportional to $\alpha B_{\text {real }}^{2}$ per pixel or, using the observed field values, $B_{\mathrm{obs}}^{2} / \alpha$. Another example is evaluation of the electric current and Ohmic dissipation in the photosphere and chromosphere (e.g. Musset et al. 2015). The current density is proportional to the spatial gradient of the magnetic field, which, in turn is proportional to $B_{\text {obs }} / \alpha$. Hence, the energy deposition rate due to Ohmic heating should be also proportional to $B_{\mathrm{obs}}^{2} / \alpha$. (However, in this case energy dissipation would also depend on actual sizes of the small-scale magnetic elements, electric resistivity and other parameters). Thus, ignoring the unresolved field structure (i.e. assuming $\alpha=1$ ) would lead to underestimation of the photospheric magnetic energy and of the Ohmic heating by factor of $1 / \alpha$. Therefore, evaluating the intrinsic magnetic field (or the filling factor) is one of the essential problems in interpreting magnetographic observations.

The most comprehensive method of the magnetic filling factor (or intrinsic magnetic field) diagnostics is the Stokes inversion (see e.g. Socas-Navarro 2001; del Toro Iniesta \& Ruiz Cobo 2016, for review). However, this method is computationally expensive 
and is usually applied to relatively small photospheric areas, consisting of up to $10^{3}-10^{4}$ pixels (e.g. Kalewicz \& Bommier 2019; Kuckein 2019). Hence, it cannot be realistically used to analyse big photospheric area, such as a large active region $\left(10^{4}-10^{6}\right.$ pixels $)$, or the whole Sun. Fast, "on-the-fly" diagnostics of big areas is usually done using simplified "proxy" methods, such as the magnetic line ratio(MLR) method. With the fast development of the Stokes invertion technics and available computational powers, these proxy methods are likely to become obsolete within 10-20 years. However, presently, they remain essential.

The MLR is the most commonly used proxy method for fast intrinsic magnetic field diagnostics in magnetometric observations. It is based on a comparison of the field values observed with two spectral lines $\left(B_{\mathrm{obs}, 1}\right.$ and $\left.B_{\mathrm{obs}, 2}\right)$ having very close formation depths but different magnetic sensisitivities (i.e. different Lande factors, $g_{1}$ and $g_{2}$ ). The magnetographic signal (Stokes $V$ amplitude) is not directly proportional to the magnetic field due to Zeeman saturation effect and, hence, it is normally possible to relate the $B_{\mathrm{obs}, 1} / B_{\mathrm{obs}, 2}$ ratios to the intrinsic field values (e.g. Stenflo 1973; Sanchez Almeida et al. 1988; Solanki 1993; Rachkovsky et al. 2005; Khomenko \& Collados 2007; Smitha \& Solanki 2017; Bellot Rubio \& Orozco Suárez 2019). The MLR is a very robust and efficient method. However, it has one obvious drawback: it requires observations of at least two lines. Furthermore, the reliability of this method is affected if the chosen lines have slightly different formation depths, or slightly different sensitivities to the temperature or density perturbations.

Gordovskyy et al. (2018) have suggested a new method for the intrinsic magnetic field (or the filling factor) requiring observations of only one line. In that method $B_{\text {real }}$ values are evaluated using the widths of Stokes $V$ peaks (Stokes $V$ width method, SVW hereafter). They compared this new method with the classical MLR method for the magneto-convective model of the photosphere and found that, at least for the widely used Fe I $6301.5 \AA$ and $6302.5 \AA$ lines (6301/6301 pair, hereafter), this method is more reliable than MLR. Thus, on average, SVW had errors smaller than MLR. However, this could be expected, taking into account that 6301.5 and 6302.5 lines have rather different formation depths (Khomenko \& Collados 2007). What is more important is that SVW did not show saturation at higher field values (1-2kG), typical for MLR.

In this paper, we explore this new method by extending it to new lines often used for solar magnetic field measurements: optical Fe I 5247.1 $\AA$ and 5250.2 $\AA$ lines (5247/5250 pair, hereafter), and near-infrared Fe I $15648.5 \AA$ and $15652.9 \AA$ pair (15648/15652 pair, hereafter). In Sect. 2, we calculate calibration curves for these lines, linking the intrinsic magnetic field values with the widths of blue peak of Stokes $V$ components for different Stokes $I$ widths. In Sect. 3, we test the reliability of this method using the synthetic profiles of the considered lines obtained from magnetohydrodynamic (MHD) magnetoconvective simulations of the photosphere.

\section{Synthetic Stokes profiles and calibration curves}

In order to understand the effect of the magnetic field and thermodynamic conditions in the photosphere on the Stokes profiles, we calculate profiles of the six chosen spectral lines for different vertical magnetic field strengths $B_{z}$ and isotropic micro-turbulent velocities $V_{\text {mic }}$. We consider a plane-parallel model of the photosphere with the normal direction $(Z)$ parallel to the LOS. The magnetic field strength, determining the Zeeman effect, is varied
Table 1. Atomic parameters of the six considered lines.

\begin{tabular}{llllll}
\hline \hline$\lambda, \AA$ & $g$ & $\chi, \mathrm{eV}$ & $\log (g f)$ & Lower term & Upper term \\
\hline 5247.1 & 2.00 & 0.087 & -4.97 & $5 \mathrm{D} 2$ & $7 \mathrm{D} 3$ \\
5250.2 & 3.00 & 0.121 & -4.96 & $5 \mathrm{D} 0$ & $7 \mathrm{D} 1$ \\
6301.5 & 1.67 & 3.654 & -0.72 & $5 \mathrm{P} 2$ & $5 \mathrm{D} 2$ \\
6302.5 & 2.50 & 3.686 & -1.24 & $5 \mathrm{P} 1$ & $5 \mathrm{D} 0$ \\
15648.5 & 3.00 & 5.426 & -0.043 & $7 \mathrm{D} 5$ & $7 \mathrm{D} 4$ \\
15652.9 & 1.53 & 6.246 & -0.675 & $7 \mathrm{D} 1$ & $7 \mathrm{D} 1$ \\
\hline
\end{tabular}

Notes. Columns are: wavelength, Lande factor, excitation potential of the lower level, transition probability, lower and upper term configurations.

in the range $0-2 \mathrm{kG}$, while the micro-turbulent velocities, affecting the line width, were varied between 0 and $1 \times 10^{5} \mathrm{~m} \mathrm{~s}^{-1}$, which is the Alfven speed for the magnetic field of $1 \mathrm{kG}$ at the photospheric level. These values are set constant with depth. All other parameters (density, temperature, chemical composition and ionisation) vary with depth according to the VAL C model (Vernazza et al. 1973).

The Stokes profiles are calculated using the NICOLE code (Socas-Navarro et al. 2000; Socas-Navarro 2011). The atomic parameters for the chosen lines are shown in Table $1^{1}$. The profiles are calculated for the $64 \times 64$ parametric grid of $\left[B, V_{\text {mic }}\right]$. The obtained profiles are used to measure the required parameters: Stokes $I$ width $W_{I}$ (its full width at half maximum), Stokes $V$ amplitude $V$ (amplitude of Stokes $V$ blue wing normalised on the Stokes $I$ intensity of the continuum), Stokes $V$ width $W_{V}$ (the full width of Stokes $V$ blue wing at its half maximum), and the splitting between centres of mass of the $I+V$ and $I-V$ profiles, $\Delta \lambda_{H}$ (Zeeman splitting, hereafter) (Fig. 1). Then, our parametric grid $\left[B, V_{\text {mic }}\right]$ is transformed to $\left[B, W_{I}\right]$, making it possible to link observable parameters $-W_{I}, V, W_{V}, \Delta \lambda_{H}$ with the magnetic field characteristics.

Increase of magnetic field affects three observables: $V, \Delta \lambda_{H}$, and $W_{V}$ show noticeable increase. Because the $V$ values for lines with higher Lande factor $g$ saturate faster than in the lines with lower $g$, the MLRs $R_{\text {MLR }}=V_{\text {lower } g} / V_{\text {higher } g}$ also increase. Importantly, these parameters are proxies for different magnetic field characteristics. The Stokes $V$ amplitude $V$ and related to it Zeeman splitting $\Delta \lambda_{H}$ are defined by the spatially-average magnetic field (or a magnetic flux per observational pixel), while the MLR $V_{\text {lower } g} / V_{\text {higher } g}$ and Stokes $V$ width $W_{V}$ are proxies of the intrinsic magnetic field strength (see Figs. 2-4 in Gordovskyy et al. 2018).

Let us consider the behaviour of the calibration curves, $R_{\mathrm{MLR}}(B)$ and $W_{V}(B)$, for different line widths $W_{I}$. These parameters are plotted in Fig. 2.

The calibration curves for MLR are very similar to those calculated in previous studies (e.g. Khomenko \& Collados 2007; Smitha \& Solanki 2017). It can be seen that both characteristics, MLR and SVW, are proxies of $B$. However, the non-saturation ranges, where they can be effectively used for $B$ estimations, and their sensitivities to the turbulent broadening are very different. As can be expected, in each pair, the line with lower Lande factor saturates at higher $B$ values. For this reason, we will compare SVW calibration curves of the lower $g$ lines with the MLR calibration curves in each pair.

The MLR curves for the 5247/5250 pair (Fig. 2c) can be used in the range from about $100-200 \mathrm{G}$ to $1100-1800 \mathrm{G}$, depending

http://www.nist.gov/pml/data/asd.cfm 
M. Gordovskyy et al.: Using Stokes $V$ widths for the photospheric magnetic field diagnostics
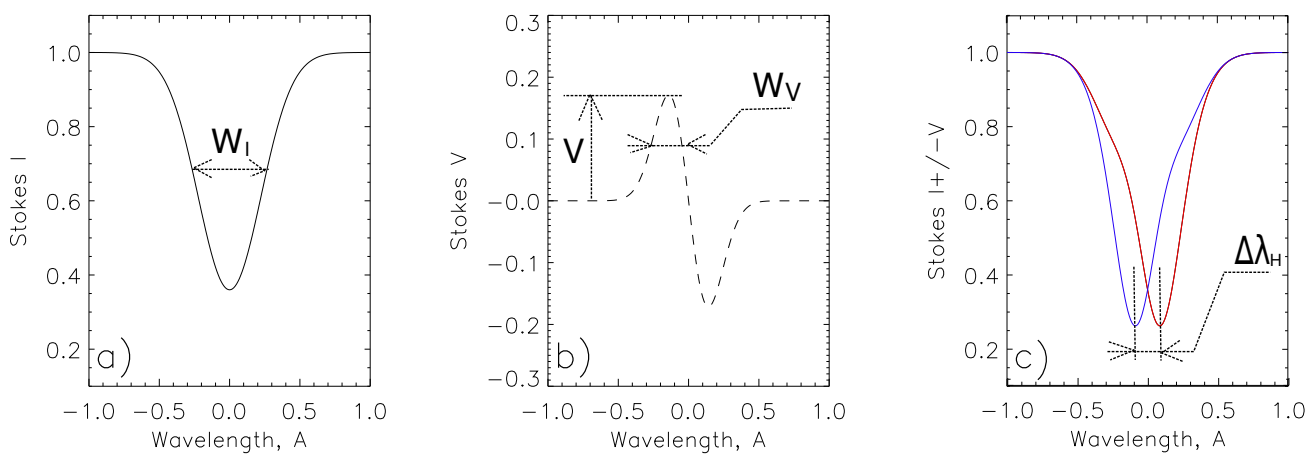

Fig. 1. Sketch of line profiles, showing Stokes $I$ profile (panel a), Stokes $V$ (panel b), and Stokes $I \pm V$ profiles (panel $c$ ), indicating the parameters used in this study.
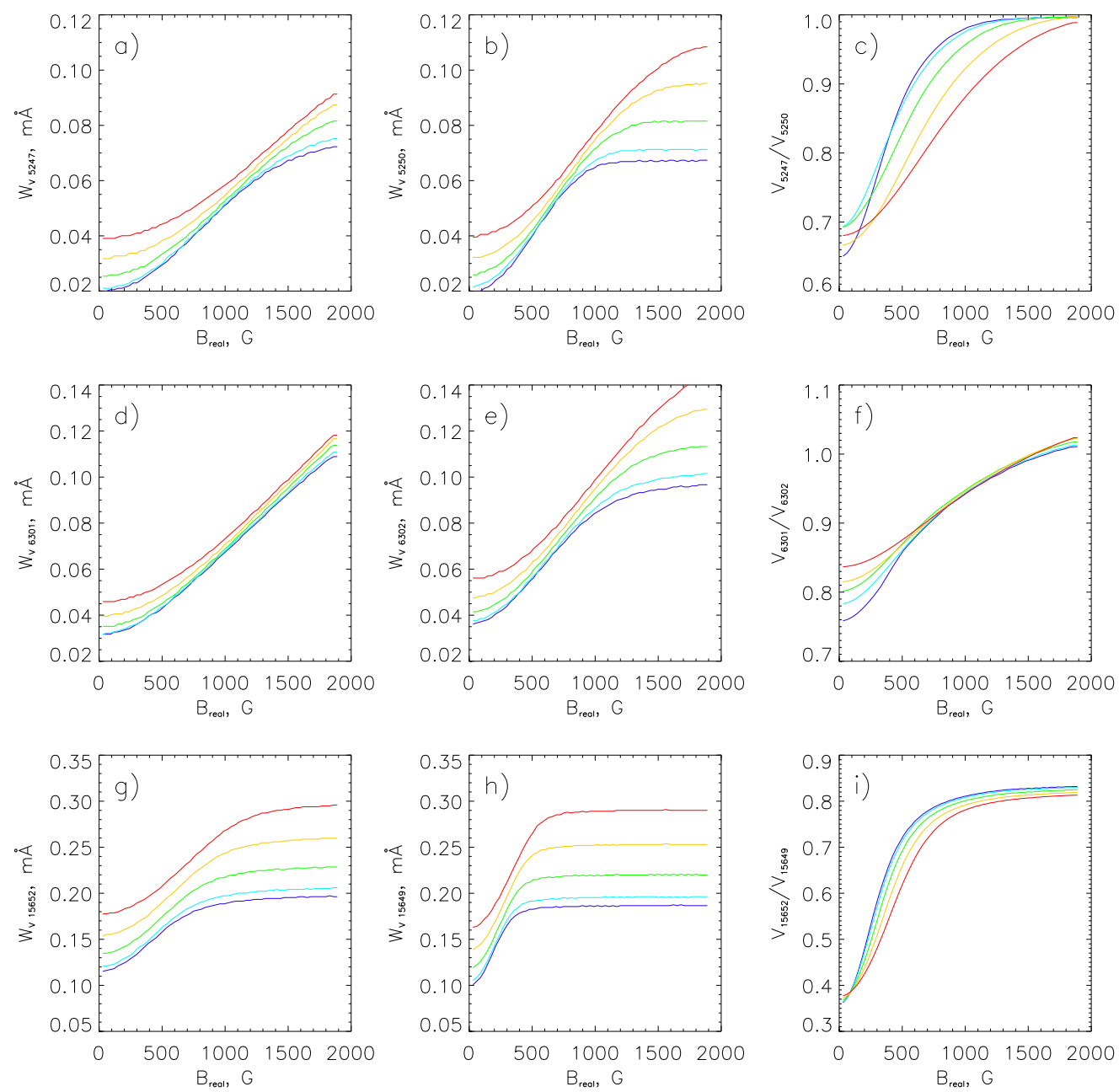

Fig. 2. Calibration curves showing Stokes $V$ width and MLR values as functions of the intrinsic field $B$ for different turbulent velocities, which determine $W_{I}$. The turbulent velocities vary from 0 (blue lines) to $20 \mathrm{~km} \mathrm{~s}^{-1}$ (red lines). Panels $a-c, d-f$ and $g-i$ correspond to $5247 / 5250,6301 / 6302$ and 15652/15649 pairs, respectively. The left column (panels $a$, $d$ and $g$ ) show Stokes $V$ widths for lines with lower Lande factors $g$, the middle column (panels $b$, $e$ and $h$ ) show Stokes $V$ widths for lines with higher Lande factors $g$, and the right column (panels $c$, fand $i$ ) shows MLR values.

on the Stokes $I$ profile width. The wider the line, the higher is the $B$ value when MLR saturates, because the condition for $V(B)$ saturation is $\Delta \lambda_{H}>W_{I}$. SVW for the 5247 line can be used from 200-300 G (Fig. 2a,b), however, it saturates at larger values of $B$, 1700-2200 G, depending on the Stokes $I$ width.

The 6301/6302 pair appears to have widest range of applicability, compared to the two other pairs. The SVW calibration curves for this pair show no saturation within the considered range, while the MLR calibration curves show very mild saturation around $2 \mathrm{kG}$. Furthermore, both MLR and SVW calibration curves for this pair show very weak dependence on the widths $W_{I}$, which, in principle, should result in smaller errors, because the error of $W_{I}$ measurements is unimportant.

The MLR for the $15648 / 15652$ pair (Fig. 2i) has a smaller range of applicability, it saturates at lower $B$ values, simply because $W_{I}$, which is determined by the Doppler effect, is 
proportional to $\lambda$, while Zeeman splitting is $\Delta \lambda_{H} \sim \lambda^{2}$. Thus, MLR for this pair can be used between 0 and 700-800 G, depending on the profile width $W_{I}$. Again, the SVW range for the 15652 line is bigger (Figs. $2 \mathrm{~g}, \mathrm{~h}$ ), it can be used from $\sim 200 \mathrm{G}$ to $800-1300 \mathrm{G}$, depending on $W_{I}$.

Therefore, based purely on the analysis of MLR and SVW calibration curves the 6301/6302 pair appears to be best both for MLR and SVW analysis, compared to the two other pairs, 5247/ 5250 and 15648/15652. However, these calibration curves are calculated using simplified photospheric models with verticallyuniform magnetic field $B$ and zero macroscopic LOS velocities $V_{\mathrm{LOS}}$. Presence of vertical gradients of $B$ (which can be very substantial in the photosphere) and Doppler shifts due to $V_{\text {LOS }}$ can substantially affect the Stokes profiles and, hence, results of the MLR and SVW analysis. For instance, it is well known that the MLR method for the 6301/6302 pair can yield substantial errors in the presence of LOS field gradient due to the substantial difference in the line formation depths. Therefore, it is necessary to test the SVW and MLR methods for the three considered line pairs using a realistic magneto-convective model of the photosphere.

The calibration curves are used to create tabulated functions (arrays) of $B_{\text {real } i j}=f\left(R_{\mathrm{MLR}}, W_{I}\right)$ and $B_{\text {real } i j}=f\left(W_{V}, W_{I}\right)$, which can be directly applied to the observational data in order to estimate the intrinsic field values. It should be noted that it can be done only to limited ranges of $R_{\mathrm{MLR}}, W_{V}$ and $W_{I}$, where these functions are increasing monotonically in respect of both input parameters. Therefore, this calibration can only be applied to pixels within these ranges.

\section{Testing MLR and SVW methods using an MHD model}

Now let us compare the two methods using one of the highresolution MHD models of magneto-convection. This threedimensional models has been developed using the MURAM code (see Schussler et al. 2003; Shelyag et al. 2004; Vogler et al. 2005). Simulations have been performed using a box with a uniform grid of $960 \times 960 \times 400$ elements with horizontal step of $5.5 \mathrm{~km}$ and vertical step of $5 \mathrm{~km}$. The upper boundary of the domain corresponds to the temperature minimum level. The model consists of two stages: it starts with simulations of convection in the initially plane-parallel photosphere corresponding to the VAL model. Then, a uniform vertical magnetic field is added, and simulations are run for about five convective timescales (corresponding to $50 \mathrm{~min}$ of physical time).

The Stokes profiles of selected lines for this model have been calculated using the NICOLE code (see description in Sect. 2). In order to get magnetograms with limited spatial resolution, we degrade the Stokes $I$ and $V$ cubes corresponding to this MHD model using the same methodology as in Gordovskyy et al. (2018). The size of "degraded" pixels is approximately 110 $150 \mathrm{~km}(0.15 \operatorname{arcsec})$. The magnetic field taken from the MHD model, magnetic field measured using Zeeman effect with reduced spatial resolution, and corresponding magnetic filling factor are shown in Fig. 3.

Obviously, in reality the magnetic field in each degraded pixel has not a single value of intrinsic field but a range of values (although, this range is rather narrow due to magnetoconvective collapse, see discussions in Vogler et al. 2005 and Gordovskyy et al. 2018). Therefore, we define the characteristic filling factor for each pixel using the definition in Gordovskyy et al. (2018).

Figure 4 compares the actual (i.e. taken from the MHD model) values of the intrinsic magnetic field with values evaluated using
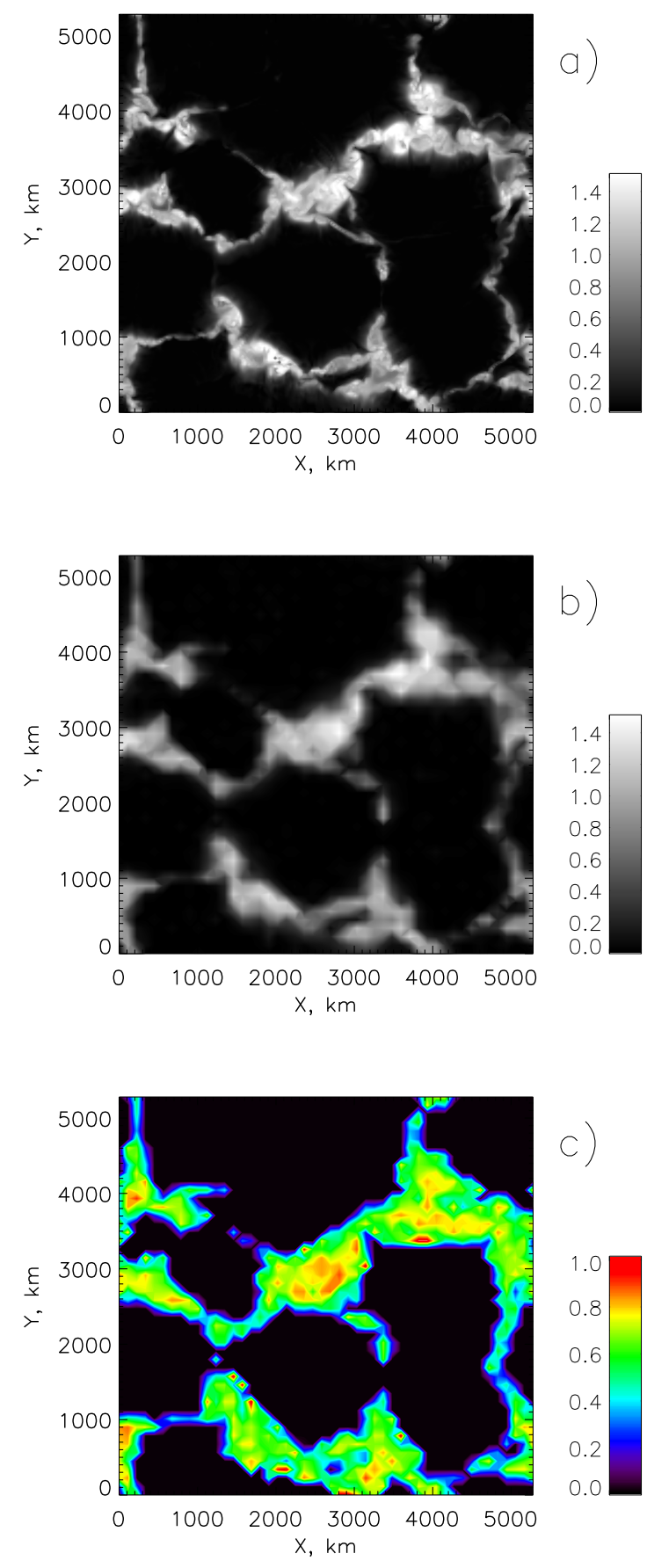

Fig. 3. Magnetic field taken from MHD simulations: panel $a$ : line-ofsight magnetic field (in $\mathrm{kG}$ ) with original resolution $(5.5 \mathrm{~km}$, please note, that the plots does not reflect the full resolution), panel $b$ : lineof-sight magnetic field (in $\mathrm{kG}$ ) measured using Zeeman splitting in Fe I 6301 A line with reduced spatial resolution $(110 \mathrm{~km})$, panel $c$ : magnetic filling factor corresponding to the spatial resolution of $110 \mathrm{~km}$. The magnetic filling factor is shown only for $\left|B_{\text {obs }}\right|$ field values greater than $100 \mathrm{G}$.

the MLR and SVW methods. For SVW estimations we use the line with lower Lande factor $g$ in each pair. For the analysis we only use pixels where the observed field values (i.e. the field values measured using the degraded Stokes cubes) are above $100 \mathrm{G}$. Obviously, all calibration functions derived in Sect. 2 have limited applicability ranges (in terms of MLR, SVW and $W_{I}$ values). Therefore, some "observed" pixels with values outside of these ranges cannot be analysed. Furthermore, we do not analyse 

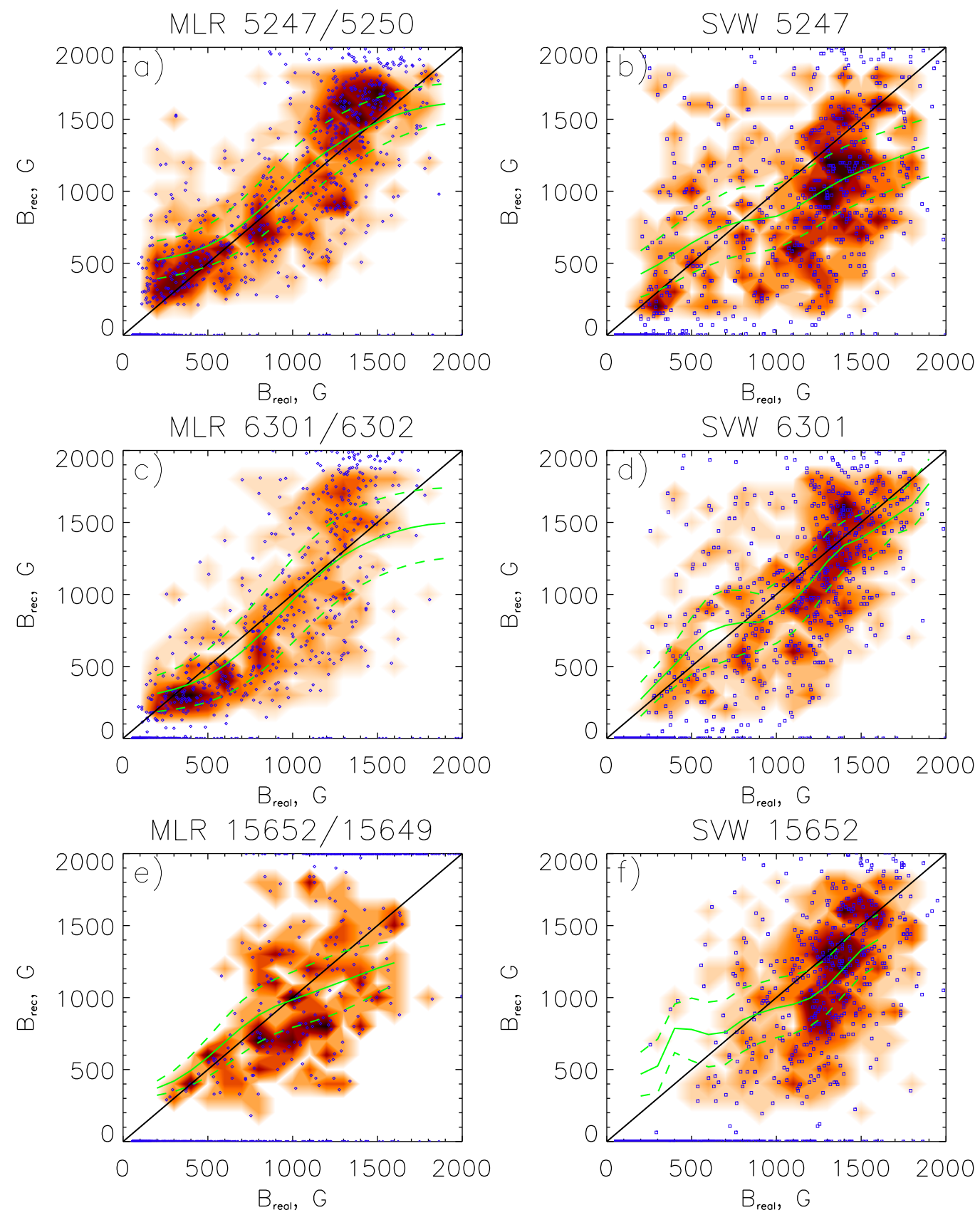

Fig. 4. Values of intrinsic field calculated using MLR (left panels) and SVW (right panels) methods compared with the actual field values taken from the model. Panels $a, b ; c, d$ and $e, f$ correspond to the 5247/5250, 6301/6302 and 15652/15648 pairs, respectively. Solid green lines denote sliding average values (calculated with the $150 \mathrm{G}$ windows); dashed green lines denote $1 \sigma$ deviation from the sliding average values.

pixels showing abnormal spectral profiles (such as multi-lobe or extremely asymmetric Stokes $V$ profiles). All these failed pixels are assigned default $B_{\text {rec }}=0$ value. That is why all panels in Fig. 4 show numerous pixels at the $B_{\text {rec }}=0$ axis. The number of failed pixels is apprximately $10 \%$ of the total pixel number. However, their proportion might be bigger if these methods are applied to the photosphere in active regions, with more fragmented velocity field, higher LOS velocity gradients, stronger turbulence and other factors.

The MLR method provides relatively good accuracy for estimations made using the 5247/5250 pair (Fig. 4a). In the interval $500-1500 \mathrm{G}$ the typical systematic error is $\sim 100 \mathrm{G}$. However, below $500 \mathrm{G}$ this method seems to overestimate the intrinsic field, and above 1600-1700 G it shows saturation, as predicted by the calibration (Fig. 2). The point spread $\sigma$ is very moderate, varying between $200-300 \mathrm{G}$ in the $200-1800 \mathrm{G}$ interval. In contrast, the SVW estimations using the 5247 line are much less reliable (Fig. 4b): the point spread $\sigma$ is around $300-400 \mathrm{G}$, and there is a substantial systematic error, with the intrinsic field in the range $1000-1500 \mathrm{G}$ being underestimated by $300-500 \mathrm{G}$.

The SVW method with the 6301 line appears to be as reliable as MLR with 5247/5250 pair (Fig. 4d), the estimations reveal typical point spread of around $200-250 \mathrm{G}$ and the systematic error between 100 and $200 \mathrm{G}$. Importantly, these relatively low errors are nearly constant in a wide range of field values, from 200 to $1900 \mathrm{G}$. The MLR method with the 6301/6302 pair appears to be less reliable (Fig. 4c), showing a slightly bigger spread, 250-350 G, and a noticeable systematic error: 
it seems to quickly saturate above $\sim 1500 \mathrm{G}$. Still, the MLR estimations with this pair are acceptable in the range below $1200-1300 \mathrm{G}$. This is consistent with the earlier analysis by Gordovskyy et al. (2018), although quite surprising, taking into account the difference in line formation depths in this pair (see e.g. Khomenko \& Collados 2007).

The 15648/15652 pair yields relatively good results with the MLR method in the range below 700-800 G (Fig. 4e). The intrinsic field values estimated using both the MLR and SVW methods with this pair of lines have a typical error of around $250 \mathrm{G}$. Above $1 \mathrm{kG}$ there is a substantial systematic error due to saturation, which is predicted by the calibration (Sect. 2). The intrinsic field estimations using the SVW method with the 15648 line (Fig. 4f) produces results with the point spread of $200-400 \mathrm{G}$ in addition to the systematic error of approximately the same value. More importantly, because this line pair is more sensitive both to the magnetic field variation, and thermal and turbulent line broadening, it produces fewer "usable" points, particularly below $1 \mathrm{kG}$. This is because many pixels have observed parameters outside of the acceptable parameter ranges (see Sect. 2). As the result, the estimations made with this line using the SVW method, should be taken with caution.

\section{Summary and discussion}

Our study of three pairs of spectral lines commonly used in solar spectropolarimetry shows that all three pairs, in principle, can be used for the intrinsic magnetic field estimations. However, they provide different accuracy and have very different validity ranges.

The 5247/5250 pair can be used both for MLR and SVW measurements. The MLR method is more reliable, yielding estimations with a typical error of 200-300 G, compared with the typical error of $300-350 \mathrm{G}$ for the SVW method. Depending on the width of the Stokes $I$ profile, the MLR method can be used for field values up to $1200-1600 \mathrm{G}$. The MLR method appears to be less reliable when applied to the "Hinode pair", $6301 / 6302$. The spread of points is bigger and there is a systematic error - above $1200 \mathrm{G}$ the magnetic field is underestimated by 300-500 G. At the same time, the SVW method works well: it provides the intrinsic field estimations with the accuracy of about $150-250 \mathrm{G}$ in the range from $200 \mathrm{G}$ to at least $1800 \mathrm{G}$. The nearinfrared pair $15648 / 15652$ provides relatively accurate results, but only for low fields, below 700-800 G. This is not surprising, since the calibration curves show that this pair quickly saturates above $\sim 700 \mathrm{G}$.

Overall, our analysis shows that the MLR method with the $5247 / 5250$ pair and the SVW method with the 6301 line provides the most reliable estimations because they do not produce large systematic errors. It should be noted, however, that the analysis is done for one, randomly selected MHD model. Both methods need to be tested further using a number of MHD models of photospheric magneto-convection with different initial magnetic field and different atmospheric structure.

Of course, the reliability of the considered methods depends on the calibration. The calibration used in this and other similar studies (see Khomenko \& Collados 2007; Smitha \& Solanki 2017; Gordovskyy et al. 2018) is based on the vertically-uniform magnetic field. Taking into account that the magnetic field in the photosphere is expected to have substantial vertical gradients, this calibration may result in significant systematic error. This can be overcome by constructing the calibration curves using the
MHD models of magneto-convection in the photosphere, similar to those used for testing in this and previous studies.

The main conclusion is that, generally, the SVW method is as good as MLR. The main drawback of the SVW method is that it requires resolved Stokes profiles with resolution of $20-30 \mathrm{~m} \AA$ in the green area and $40-50 \mathrm{~m} \AA$ in the near-infrared range. At the same time, the SVW method has an important advantage over MLR: it requires only one spectral line and, hence, is not affected by the line formation difference. The latter is practically significant because a number of widely-used instruments observe only one spectral line, or observe a pair not suitable for using the MLR method (such as Hinode/SOT).

Using the SVW method for fast intrinsic magnetic field diagnostics requires spectropolarimentric observations with large field of view and reasonably good spectral resolution, but only moderate spatial resolution. Forthcoming DKIST (Tritschler et al. 2016) and future EST (Jurák et al. 2019) solar telescopes are expected to provide this type of data in grating spectroscopy mode. The Stokes $V$ Width is a new method and we plan additional tests of it involving high-resolution MHD models in order to investigate the influence of non-LTE effects on the reliability of this method, and by using actual spectropolarimetric data to further compare SVW and MLR.

Acknowledgements. M.G. and P.K.B. are funded by Science and Technology Facilities Council (UK), grant ST/P000428/1. V.G.L. is funded by Taras Shevchenko National University of Kyiv, project 19BF023-03. Simulations have been performed using DiRAC Data Centric system at Durham University, operated by the Institute for Computational Cosmology on behalf of the STFC DiRAC HPC Facility.

\section{References}

Bellot Rubio, L., \& Orozco Suárez, D. 2019, Liv. Rev. Sol. Phys., 16, 1

De Wijn, A. G., Stenflo, J. O., Solanki, S. K., \& Tsuneta, S. 2009, Space Sci. Rev., 114, 275

Frazier, E. N., \& Stenflo, J. O. 1972, Sol. Phys., 27, 330

Gordovskyy, M., \& Lozitsky, V. G. 2014, Sol. Phys., 289, 3681

Gordovskyy, M., Shelyag, S., Browning, P. K., \& Lozitsky, V. G. 2018, A\&A, 619, A164

Jang, H. 2016, Sol. Phys., 291, 3501

Jurák, J., Collados, M., Leenaarts, J., van Noort, M., \& Schlichenmaier, R. 2019, AdSpR, 63, 1389

Kalewicz, T., \& Bommier, V. 2019, A\&A, 629, A138

Kuckein, C. 2019, A\&A, 630, A139

Khomenko, E., \& Collados, M. 2007, ApJ, 659, 1726

Musset, S., Vilmer, N., \& Bommier, V. 2015, A\&A, 580, A106

Rachkovsky, D. N., Tsap, T. T., \& Lozitsky, V. G. 2005, A\&A, 26, 435

Sanchez Almeida, J., Solanki, S. K., Collados, M., \& del Toro Iniesta, J. C. 1988, A\&A, 196, 266

Schussler, M., Shelyag, S., Berdyugina, S., Vgler, A., \& Solanki, S. K. 2003, ApJ, 597, L173

Shelyag, S., Schussler, M., Solanki, S. K., Berdyugina, S. V., \& Vogler, A. 2004, A\&A, 427, 335

Smitha, H.N., \& Solanki, S.K. 2017, A\&A, 608, A111

Socas-Navarro, H. 2001, ASP Conf. Ser., 236, 487

Socas-Navarro, H. 2011, A\&A, 529, A37

Socas-Navarro, H., Trujillo Bueno, J., \& Ruiz Cobo, B. 2000, ApJ, 544, 1141

Solanki, S. K. 1993, Space Sci. Rev., 63, 1

Stenflo, J. O. 1973, Sol. Phys., 32, 41

Stenflo, J. O. 2011, A\&A, 529, A42

del Toro Iniesta, J. C., \& Ruiz Cobo, B. 2016, Liv. Rev. Sol. Phys., 13, 4

Tritschler, A., Rimmele, T. R., Berukoff, S., et al. 2016, Astron. Nach., 337, 1064

Vernazza, J. E., Avrett, E. H., \& Loeser, R. 1973, ApJ, 184, 605

Vogler, A., Shelyag, S., Schussler, M., Cattaneo, F., Emonet, T., \& Linde, T. 2005, A\&A, 429, 335

Welsch, B. T. 2014, PASJ, 67, 18

Wiegelmann, T., \& Sakurai, T. 2012, Liv. Rev. Sol. Phys., 9, 5 